\title{
ALOX12 polymorphisms are associated with fat mass but not peak bone mineral density in Chinese nuclear families
}

\author{
W-J Xiao, J-W He, H Zhang, W-W Hu, J-M Gu, H Yue, G Gao, J-B Yu, C Wang, Y-H Ke, W-Z Fu \\ and Z-L Zhang
}

Department of Osteoporosis, Metabolic Bone Disease and Genetic Research Unit, Shanghai Jiao Tong University Affiliated Sixth People's Hospital, Shanghai, People's Republic of China

Objective: Arachidonate 12-lipoxygenase (ALOX12) is a member of the lipoxygenase superfamily, which catalyzes the incorporation of molecular oxygen into polyunsaturated fatty acids. The products of ALOX12 reactions serve as endogenous ligands for peroxisome proliferator-activated receptor $\gamma$ (PPARG). The activation of the PPARG pathway in marrow-derived mesenchymal progenitors stimulates adipogenesis and inhibits osteoblastogenesis. Our objective was to determine whether polymorphisms in the ALOX12 gene were associated with variations in peak bone mineral density (BMD) and obesity phenotypes in young Chinese men.

Methods: All six tagging single-nucleotide polymorphisms (SNPS) in the ALOX12 gene were genotyped in a total of 1215 subjects from 400 Chinese nuclear families by allele-specific polymerase chain reaction. The BMD at the lumbar spine and hip, total fat mass (TFM) and total lean mass (TLM) were measured using dual-energy X-ray absorptiometry. The pairwise linkage disequilibrium among SNPs was measured, and the haplotype blocks were inferred. Both the individual SNP markers and the haplotypes were tested for an association with the peak BMD, body mass index, TFM, TLM and percentage fat mass (PFM) using the quantitative transmission disequilibrium test (QTDT).

Results: Using the QTDT, significant within-family association was found between the rs2073438 polymorphism in the ALOX12 gene and the TFM and PFM $(P=0.007$ and 0.012 , respectively). Haplotype analyses were combined with our individual SNP results and remained significant even after correction for multiple testing. However, we failed to find significant within-family associations between ALOX12 SNPs and the BMD at any bone site in young Chinese men.

Conclusions: Our present results suggest that the rs 2073438 polymorphism of ALOX12 contributes to the variation of obesity phenotypes in young Chinese men, although we failed to replicate the association with the peak BMD variation in this sample. Further independent studies are needed to confirm our findings.

International Journal of Obesity (2011) 35, 378-386; doi:10.1038/ijo.2010.157; published online 10 August 2010

Keywords: ALOX12; fat mass; lean mass; peak bone mineral density; quantitative transmission disequilibrium test

\section{Introduction}

It was once thought that osteoporosis and obesity were unrelated, ${ }^{1}$ but there is accumulating evidence confirming their association in recent decades. Weight is a major determinant of bone mineral density (BMD); subjects who are overweight or obese have a relatively higher BMD, and

Correspondence: Professor Z-L Zhang, Department of Osteoporosis, Metabolic Bone Disease and Genetic Research Unit, Shanghai Jiao Tong University Affiliated Sixth People's Hospital, No. 600 Yi-Shan Road, Shanghai 200233, People's Republic of China.

E-mail: ZZL2002@medmail.com.cn

Received 16 March 2010; revised 8 June 2010; accepted 24 June 2010; published online 10 August 2010 weight loss is associated with an increased risk of osteoporotic fracture. ${ }^{2,3}$ With aging, bone mass is reduced, whereas adipocyte volume in bone marrow is increased. ${ }^{4}$ Both osteoporosis and obesity have strong genetic components, as the heritability of obesity is estimated at $40-60 \%,{ }^{5}$ and $60-80 \%$ of the variance in BMD is genetically determined. ${ }^{6}$ In addition, both osteoblasts and adipocytes are derived from mesenchymal stem cells. Extensive data have shown that both adipogenesis and osteogenesis share multiple common genetic factors and signaling pathways, such as vitamin D receptor, peroxisome proliferator-activated receptor $\gamma$ (PPARG), transforming growth factor- $\beta$ and Wnt pathways. ${ }^{1,7,8}$. Meanwhile, adipocytes can secrete estrogen, leptin and inflammatory cytokines to influence bone metabolism. ${ }^{9}$ Therefore, 
exploring the interface between bone and fat at the molecular and genetic levels may improve our understanding of osteoporosis and obesity, which may lead to new interventions and treatments.

Body weight is determined mostly by fat, muscle and bone mass. Body composition assessment by dual-energy X-ray absorptiometry (DXA) is a readily accessible, inexpensive and non-invasive method. DXA detects total body mineral mass, total fat mass (TFM) and total lean mass (TLM). The relationships between bone mass and TFM and TLM have been examined, although the results are controversial. ${ }^{10-12}$ Studies have also shown several genomic regions that influence both obesity and osteoporosis. ${ }^{13,14}$ Although various candidate genes have been identified that may contribute to BMD, far less is known about genes affecting obesity phenotypes such as BMI, TLM and TFM.

Recently, Klein et al. ${ }^{15}$ suggested that mouse arachidonate 15-lipoxygenase (ALOX15) could negatively regulate peak bone mass. Compared with control mice, ALOX15 knockout mice have a higher BMD, and pharmacologic inhibitors of ALOX15 improve the BMD and bone strength in a model of osteoporosis. It has also been reported that overexpression of human ALOX15 in transgenic rabbits protects against bone loss. ${ }^{16}$ However, based on the products of their lipoxygenase reactions, mouse ALOX15 is functionally more similar to human ALOX12. ${ }^{17,18}$ Therefore, human ALOX12 might represent a strong candidate gene for susceptibility to osteoporosis. In fact, a positive correlation between ALOX12 gene polymorphisms and BMD in human beings has been established by previous studies, ${ }^{19-21}$ although all the studies were limited to Caucasian samples. So far, no such data have been reported for an Asian population. Moreover, most previous studies of candidate genes have been performed in women, with only one study focusing on men.

The human ALOX12 gene is composed of 14 exons and 13 introns, mapping to chromosome $17 \mathrm{p} 13$, a region that is thought to be associated with body mass index (BMI) (http:// obesitygene.pbrc.edu). Furthermore, ALOX12 has an important function in obesity-related complex phenotypes, including hypertension, diabetes, atherosclerosis and insulin secretion. ${ }^{22-24}$ However, the relationship between ALOX12 and obesity has never been studied. In this study, we performed family-based-association analyses on all six tagging single-nucleotide polymorphisms (SNPs) in the ALXO12 gene using the quantitative transmission disequilibrium test (QTDT) to determine whether SNPs and haplotypes in $A L X O 12$ were associated with peak BMD variations and obesity-related phenotypes in a large sample of Chinese males in nuclear families.

\section{Materials and methods}

\section{Subjects}

We recruited 1296 individuals from 427 Chinese nuclear families with male offspring from 2004 to 2007 . The average family size was 3.03, as 402 and 25 families had 1 and 2 children, respectively. Every study subject completed a questionnaire concerning age, sex, medical history, family history, marital status, physical activity, alcohol use, diet habits and smoking history. All male offspring were healthy. The following criteria were used to exclude individuals from the study: (1) serious effects from cerebrovascular disease; (2) diabetes mellitus; (3) chronic renal disease; (4) serious chronic liver disease or alcoholism; (5) significant chronic lung disease; (6) corticosteroid therapy at pharmacologic levels for $>3$ months; (7) treatment with anticonvulsant therapy for $>6$ months; (8) evidence of other metabolic or inherited bone diseases such as hyper- or hypoparathyroidism, Paget's disease of the bone, osteomalacia, osteogenesis imperfecta or others; (9) rheumatoid arthritis or collagen disease; (10) recent major gastrointestinal disease (within the past year) such as peptic ulcer, malabsorption, chronic ulcerative colitis, regional enteritis or any significant chronic diarrhea state; (11) significant disease of any endocrine organ that would affect bone mass; (12) hyperthyroidism and (13) any neurological or musculoskeletal condition that would be a non-genetic cause of low bone mass.

All study subjects belonged to the Chinese Han ethnic group. All were residents of Shanghai City, located on the mid-eastern coast of China. The study was approved by the ethics committee of the Shanghai Jiao Tong University Affiliated Sixth People's Hospital. All subjects signed informed consent documents before entering the study.

\section{$B M D$ and body composition measurements}

The BMD of the lumbar spine 1-4, the left proximal femur (including the femoral neck and the total hip), as well as TFM and TLM were measured by DXA on a GE-LUNAR Prodigy (Lunar Corp., Madison, WI, USA) in fan-beam mode. All subjects were measured for BMD, and all sons were also measured for TFM and TLM. The machine was calibrated daily, and the coefficient of variability values of the DXA measurements (which were obtained from 15 individuals repeated three times) were $1.39 \%$ for the lumbar spine, $2.22 \%$ for the femoral neck and $0.70 \%$ for the total hip. The coefficient of variabilities were 1.18 and $3.72 \%$ for TLM and LFM, respectively. The long-term reproducibility of our DXA data during the trial, based on weekly repeated phantom measurements using standardized equipment, was $0.45 \%$. BMI is defined as body weight/height ${ }^{2}\left(\mathrm{~kg} \mathrm{~m}^{-2}\right)$. The percentage fat mass (PFM) is the ratio of TFM to body weight.

\section{Genotyping}

SNPs located within the ALOX12 gene were selected from the NCBI LocusLink (http://Ncbi.nlm.nih.gov/LocusLink) and HapMap (http://hapmap.org). Polymorphisms spanning the ALOX12 gene were selected from the SNPs resource based on the estimated pairwise linkage disequilibrium (LD), $r^{2}$, between all common SNPs. The algorithm bins SNPs whose 
pairwise LD exceeds a threshold $r^{2}$ of 0.8 , tagging SNPs are selected from each bin to constitute a minimal set of highly informative markers, whereas minimizing redundant data. We selected SNPs on the basis of the following criteria: (1) degree of heterozygosity (minor allele frequencies $>0.10$ ) and (2) classification as tagging SNPs. In total, six SNPs in the ALOX12 gene were selected: rs2073438, rs2292350, rs312470, rs434473, rs1235805 and rs312462. The study subjects were genotyped for all six SNPs using the TaqMan allelic discrimination assay (Applied Biosystems, Foster City, CA, USA), whereas primer and probe sequences were optimized using the SNP assay-by-design service of Applied Biosystems. Reactions were performed on the Mx3000P Real-Time PCR System (STRATAGENE, La Jolla, CA, USA). One allelic probe was labeled with FAM dye and the other with HEX dye. Genomic DNA (20 ng) was amplified on 96-well plates in the presence of a $1 \times$ TaqMan probe assay and $1 \times$ TaqMan Universal PCR Master Mix (Applied Biosystems).

\section{LD and haplotype analyses}

Haplotypes were constructed from the population genotype data by the algorithm of Stephens using the Phase program, version 2.0.2. Significant levels of $\mathrm{LD}$ between the markers of this gene were assessed based on the observed haplotype and allele frequencies using Haploview 3.2. ${ }^{25}$ We examined Lewontin's $D$ and LD coefficient $r^{2}$ between all pairs of biallelic loci. The frequencies of genotypes and haplotypes were calculated using the unrelated parents of nuclear families.

\section{Statistical analyses}

The allele frequencies were estimated by gene counting. Hardy-Weinberg equilibrium was tested by a $\chi^{2}$ goodnessof-fit statistic. To ensure unrelated individual samples, only genotype data from the parents of each nuclear family were used in the statistical analysis. The heritability estimate was performed using a linear regression of the parents' mean value and the offspring's value of every phenotype (described at http://www.heritability.com). The statistical power was estimated with Piface software (version 1.65) (http:// www.math.uiowa.edu/ rlenth/Power/) on our current sample size according to the minor allele frequency of every genotype and the variation of the BMD and obesity phenotypes. The QTDT program using the orthogonal model was used to test for population stratification, linkage and within-family association between SNPs and haplotypes and BMD phenotypes, TLM, TFM and BMI. The QTDT software package is available online (http://www.sph.umich.edu/csg/ abecasis/QTDT/). This method, as implemented in the QTDT software, ${ }^{26}$ extends the trio-based TDT to quantitative trait data and uses genotype data from available siblings and parents. As in our nuclear families, all of the children were sons and the effects of parent phenotypes were excluded in the QTDT, sex was not used as a covariate to adjust the variations in the sons' bone phenotypes. The raw BMD values were adjusted by age, height and weight as covariates, and BMI, TFM and TLM were adjusted by age as covariates. As false-positive results might be generated in multiple tests, which were used in this study, permutations (1000 simulations) were performed to generate the empirical $P$-values ${ }^{26,27}$ to assess the reliability of the results. The QTDT program generates $P$-values for various tests through an asymptotic $\chi^{2}$ distribution. $P<0.05$ was considered significant for all analyses.

In unrelated sons, the differences in the BMD among the genotype and haplotype groups were tested using general linear model ANOVA (GLM-ANOVA) adjustments for the confounding variables of age, height and weight. The differences in the BMI, TFM and TLM among the genotype and haplotype groups were performed using GLM-ANOVA adjusted for age. To evaluate the associations of the TFM and TLM with the BMD, we used Pearson's correlation analyses to examine the coefficient (r) and multiple linear regression to estimate the relative contribution of the TFM and TLM to the BMD at various sites in young men. Statistical analysis was performed using SPSS, version 11.0 (SPSS, Chicago, IL, USA).

\section{Results}

Basic characteristics of the study subjects

Of the males sampled from 427 nuclear families, 15 individuals could not be genotyped because of the poor quality of the DNA sample, and 12 sons deviated from Mendelian inheritance. Ultimately, there were 400 male nuclear families composed of 1215 individuals in this study. The basic characteristics of the study subjects are shown in Table 1. As the effects of parent phenotypes were excluded in the statistical analysis (both the QTDT and ANOVA), we only obtained the TFM and TLM of sons in this study. Peak BMD is thought to be under strong genetic control. In our sample, the heritability estimates for the peak BMD in the

Table 1 Basic characteristics of the subjects (mean \pm s.d.)

\begin{tabular}{lccc}
\hline Variables & $\begin{array}{c}\text { Father } \\
(\mathrm{n}=400)\end{array}$ & $\begin{array}{c}\text { Mother } \\
(\mathrm{n}=400)\end{array}$ & $\begin{array}{c}\text { Son } \\
(\mathrm{n}=415)\end{array}$ \\
\hline Age (years) & $61.1 \pm 7.1$ & $58.4 \pm 6.3$ & $30.4 \pm 6.1$ \\
Height (cm) & $167.8 \pm 6.0$ & $155.7 \pm 5.5$ & $172.9 \pm 5.9$ \\
Weight (kg) & $69.7 \pm 9.5$ & $58.2 \pm 8.2$ & $70.7 \pm 10.8$ \\
BMI (kg m${ }^{-2}$ ) & $25.2 \pm 2.7$ & $24.0 \pm 3.1$ & $24.2 \pm 3.2$ \\
Lumbar spine BMD $\left(\mathrm{g} \mathrm{cm}^{-2}\right)$ & $1.139 \pm 0.171$ & $0.992 \pm 0.168$ & $1.138 \pm 0.137$ \\
Femoral neck BMD ( $\left.\mathrm{g} \mathrm{cm}^{-2}\right)$ & $0.892 \pm 0.132$ & $0.796 \pm 0.144$ & $0.995 \pm 0.141$ \\
Total hip BMD (g cm $\left.{ }^{-2}\right)$ & $0.958 \pm 0.138$ & $0.852 \pm 0.162$ & $1.008 \pm 0.142$ \\
TFM (kg) & - & - & $16.31 \pm 7.56$ \\
TLM (kg) & - & - & $51.43 \pm 5.76$ \\
PFM (\%) & - & - & $21.89 \pm 7.17$ \\
\hline
\end{tabular}

Abbreviations: BMD, bone mineral density; BMI, body mass index; PFM, percentage fat mass; TFM, total fat mass; TLM, total lean mass. 
spine, femoral neck and total hip were $0.565,0.702$ and 0.693 , respectively.

\section{Relationship between the BMD and the TFM and TLM in young men}

As shown in Table 2, Pearson's correlation analyses confirmed that both the TFM and TLM were positively correlated with the BMD, with correlations ranging from 0.198 to 0.508 for the lumbar spine, femoral neck and total hip BMD. According to the Pearson's coefficient ( $r$ ), the TLM was more strongly related to the BMD at a given site. To determine the relative contributions of the TLM and TFM to the BMD, we constructed multiple regression models, which were simultaneously adjusted for age and height. Both the TLM and TFM were positively correlated

Table 2 Pearson's correlation coefficient $(r)$ of the BMD with the TFM and TLM

\begin{tabular}{lccccc}
\hline Variation & $\begin{array}{c}\text { Lumbar spine } \\
B M D\left(\mathrm{~g} \mathrm{~cm}^{-2}\right)\end{array}$ & $\begin{array}{c}\text { Femoral neck } \\
B M D\left(\mathrm{~g} \mathrm{~cm}^{-2}\right)\end{array}$ & $\begin{array}{c}\text { Total hip BMD } \\
\left(\mathrm{g} \mathrm{cm}^{-2}\right)\end{array}$ & $\begin{array}{c}\text { TFM } \\
(\mathrm{kg})\end{array}$ & $\begin{array}{c}\text { TLM } \\
(\mathrm{kg})\end{array}$ \\
\hline Lumbar spine & - & - & - & - & - \\
BMD $\left(\mathrm{g} \mathrm{cm}^{-2}\right)$ & - & - & - & - & - \\
Femoral neck & 0.639 & - & - & - & - \\
BMD $\left(\mathrm{g} \mathrm{cm}^{-2}\right)$ & $(0.000)$ & - & - & - & - \\
Total hip & 0.719 & 0.922 & - & - & - \\
BMD $\left(\mathrm{g} \mathrm{cm}^{-2}\right)$ & $(0.000)$ & $(0.000)$ & - & - & - \\
Total fat & 0.243 & 0.198 & 0.242 & - & - \\
mass $(\mathrm{kg})$ & $(0.000)$ & $(0.000)$ & $(0.000)$ & - & - \\
Total lean & 0.389 & 0.470 & 0.508 & 0.367 & - \\
mass $(\mathrm{kg})$ & $(0.000)$ & $(0.000)$ & $(0.000)$ & $(0.000)$ & - \\
\hline
\end{tabular}

Abbreviations: BMD, bone mineral density; TFM, total fat mass; TLM, total lean mass. Statistical significance is indicated in parentheses with the relevant $P$-values.

Table 3 Multivariate regression analysis of the BMD against body composition components

\begin{tabular}{llcccc}
\hline BMD & \multicolumn{2}{c}{ Total lean mass } & & \multicolumn{2}{c}{ Total fat mass } \\
\cline { 2 - 3 } \cline { 5 - 6 } & $\beta$ & $R^{2}$ & & $\beta$ & $R^{2}$ \\
\hline Lumbar spine & $0.010^{* *}$ & 0.149 & & $0.005^{* *}$ & 0.056 \\
Femoral neck & $0.012^{* *}$ & 0.210 & & $0.004^{* *}$ & 0.051 \\
Total hip & $0.012^{* *}$ & 0.249 & & $0.005^{* *}$ & 0.068 \\
\hline
\end{tabular}

Abbreviations: BMD, bone mineral density; TFM, total fat mass; TLM, total lean mass. Models were adjusted for age and height. ${ }^{*} P<0.001$. with the BMD, whereas the TLM had a significantly greater effect on the BMD than the TFM per kilogram of tissue mass (Table 3).

\section{SNP genotyping and $L D$}

Six SNPs in the ALOX12 gene were genotyped; rs1235805 was excluded from further analysis because of the existence of homologous gene sequences, and too many families failed to pass the Mendelian inheritance check. The remaining five SNPs had a minor allele frequency of at least 0.15 and were in Hardy-Weinberg equilibrium (Table 4).

To gain insight into the pattern of LD between alleles at polymorphic loci, pairwise disequilibria measures $(D)$ were calculated (Figure 1). We found that each of these SNPs was in strong LD $(D>0.80)$, and one block with high LD was identified. We inferred that 19 different haplotypes were present in our population using a likelihood method based on PHASE. The most common haplotype (AGAGG) had a frequency of $31.8 \%$, and four haplotypes (AGAGG, GAAAG,

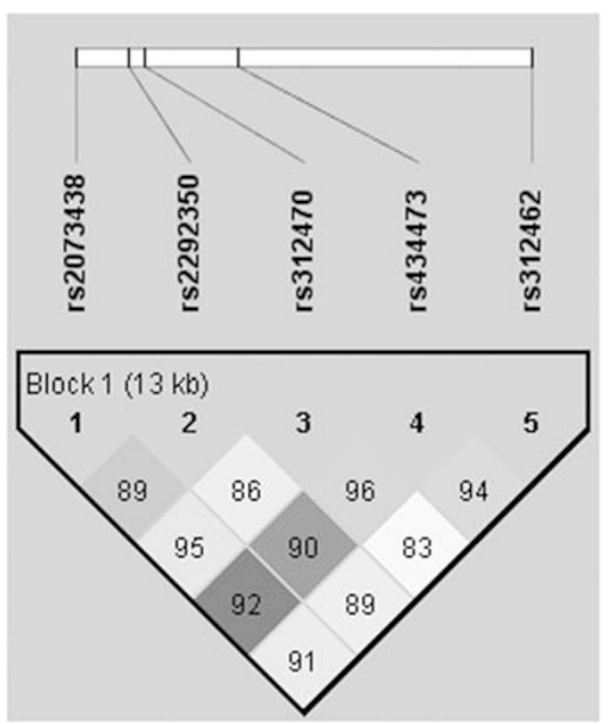

Figure 1 LD patterns for the ALOX12 gene. The increasing degree of the darkness of the cells from white to black represents the increasing strength of the LD. The values in the cells are the pairwise degree of LD indicated by $D \times 100$ when $D<1$.

Table 4 Information on the ALOX12 SNPs analyzed in this study

\begin{tabular}{|c|c|c|c|c|c|c|c|}
\hline SNP & Physical position & Location and function & Allele change & HWE P-value & MAF in European ${ }^{\mathrm{a}}$ & MAF in Asian $^{\mathrm{a}}$ & MAF in this study \\
\hline rs2073438 & 6840800 & Intron 1 & $A>C$ & 0.43 & 0.308 & 0.333 & 0.353 \\
\hline rs2292350 & 6842396 & Intron 2 & $A>G$ & 0.98 & 0.645 & 0.263 & 0.338 \\
\hline rs312470 & 6842903 & Intron 4 & $A>G$ & 0.57 & 0.045 & 0.256 & 0.159 \\
\hline rs434473 & 6845658 & Exon 8 (Asn322Ser) & $A>G$ & 0.44 & 0.467 & 0.444 & 0.494 \\
\hline rs312462 & 6854376 & Exon 14 (Leu634Leu) & $G>A$ & 0.24 & 0.133 & 0.111 & 0.151 \\
\hline
\end{tabular}

Abbreviations: HWE, Hardy-Weinberg equilibrium; MAF, minor allele frequency; SNP, single-nucleotide polymorphism. ${ }^{\mathrm{a} A c c o r d i n g}$ to public information available in the dbSNP (http://www.ncbi.nlm.nih.gov) and the HapMap (http://www.Hapmap.org) databases. 
GGGAG and GGAGA) accounted for $91.8 \%$ of the unrelated parents.

Association of the peak BMD and obesity phenotypes with SNPS in nuclear families

ANOVA and QTDT analyses. Using 400 nuclear families has a power of $>80 \%$ to test a candidate gene as a QTL, which can explain $\sim 10 \%$ of the variation in the BMD or obesity phenotypes. We investigated the association of the SNP genotypes with the BMD and obesity phenotypes using ANOVA in 400 unrelated sons randomly selected from 415 sons. Two SNPs (rs2073438 and rs312462) showed significant association with the TFM and PFM $(P<0.05)$. The sons with the AA genotype at rs2073438 had a higher adjusted TFM $(P=0.001)$ and a higher PFM $(P=0.014)$ compared with the GG genotype. This SNP contributed to 3.8 and $2.6 \%$ of the FM and PFM variation, respectively, in our sample. Individuals carrying the GG genotype at rs312462 had a higher TFM and PFM compared with GA carriers $(P=0.011$ and 0.003 , respectively). The contributions of rs312462 to the phenotypic variation of TFM and PFM were 3.0 and $3.7 \%$, respectively. However, no significant association was found between any SNP and the BMD, BMI or TLM variation in young men (Table 5).

The associations among the peak BMD, obesity phenotypes and SNPs in ALOX12 were analyzed using the QTDT for our nuclear families. As the effects of parent phenotypes were excluded in the analyses, all 400 families were included. There were 284, 273, 195, 310 and 189 informative families for the TDT analysis at rs2073438, rs2292350, rs312470, rs434473 and rs312462, respectively. We did not detect significant population stratification in our sample. The SNPs rs2073438 and rs312462 were significantly associated with the TFM and PFM in the total-association analysis $(P<0.01)$. For the within-family analysis, significant associations were found between rs2073438 and the TFM, PFM and BMI $(P=0.007,0.012$ and 0.038 , respectively), whereas rs312462 showed only marginal association with the TFM $(P=0.049)$. In the multiple parameters tests, permutations of 1000 tests were performed. However, only rs2073438 retained significant with-family associations with both the TFM and the PFM $(P=0.033$ and 0.015 , respectively). Similar to the results of the ANOVA, we failed to find significant associations

Table 5 Association of SNPs with phenotypic values in unrelated sons from single-offspring families (mean \pm s.d.)

\begin{tabular}{|c|c|c|c|c|c|c|c|c|}
\hline Genotype & $\mathrm{n}$ & $\begin{array}{l}\text { Lumbar spine } \\
\text { BMD }\left(\mathrm{g} \mathrm{cm}^{-2}\right)\end{array}$ & $\begin{array}{l}\text { Femoral neck } \\
\text { BMD }\left(\mathrm{g} \mathrm{cm}^{-2}\right)\end{array}$ & $\begin{array}{c}\text { Total hip } \\
\text { BMD }\left(\mathrm{g} \mathrm{cm}^{-2}\right)\end{array}$ & $\begin{array}{c}B M I \\
\left(k^{-2} m^{-2}\right)\end{array}$ & $\operatorname{TFM}(\mathrm{kg})$ & PFM (\%) & $\operatorname{TLM}(\mathrm{kg})$ \\
\hline \multicolumn{9}{|l|}{ rs2073438 } \\
\hline GG & 173 & $1.140 \pm 0.142$ & $1.002 \pm 0.137$ & $1.018 \pm 0.137$ & $23.5 \pm 3.5$ & $15.06 \pm 6.83$ & $20.90 \pm 7.01$ & $51.15 \pm 5.32$ \\
\hline $\mathrm{AG}$ & 180 & $1.121 \pm 0.132$ & $0.995 \pm 0.149$ & $1.013 \pm 0.143$ & $23.7 \pm 3.3$ & $16.32 \pm 6.85$ & $22.13 \pm 7.15$ & $51.37 \pm 5.99$ \\
\hline AA & 47 & $1.184 \pm 0.136$ & $0.983 \pm 0.137$ & $1.019 \pm 0.124$ & $24.1 \pm 3.1$ & $18.37 \pm 6.93^{\#}$ & $24.46 \pm 6.67^{\# \#}$ & $50.96 \pm 5.33$ \\
\hline$P$-value & & 0.076 & 0.579 & 0.696 & 0.597 & 0.001 & 0.015 & 0.918 \\
\hline \multicolumn{9}{|l|}{ rs2292350 } \\
\hline GG & 168 & $1.139 \pm 0.139$ & $0.994 \pm 0.139$ & $1.017 \pm 0.131$ & $23.7 \pm 3.3$ & $16.49 \pm 7.98$ & $21.94 \pm 6.99$ & $51.47 \pm 5.45$ \\
\hline $\mathrm{AG}$ & 183 & $1.140 \pm 0.133$ & $1.000 \pm 0.148$ & $1.020 \pm 0.149$ & $23.8 \pm 3.5$ & $16.06 \pm 7.11$ & $21.99 \pm 7.38$ & $51.06 \pm 5.86$ \\
\hline $\mathrm{AA}$ & 49 & $1.124 \pm 0.150$ & $0.989 \pm 0.119$ & $0.986 \pm 0.107$ & $22.8 \pm 2.6$ & $15.10 \pm 6.42$ & $21.16 \pm 7.18$ & $51.01 \pm 5.32$ \\
\hline$P$-value & & 0.857 & 0.802 & 0.592 & 0.171 & 0.598 & 0.843 & 0.807 \\
\hline \multicolumn{9}{|l|}{ rs312470 } \\
\hline $\mathrm{AA}$ & 278 & $1.138 \pm 0.137$ & $0.994 \pm 0.139$ & $1.011 \pm 0.132$ & $23.5 \pm 3.2$ & $16.23 \pm 7.87$ & $21.83 \pm 7.53$ & $51.31 \pm 5.54$ \\
\hline AG & 111 & $1.146 \pm 0.139$ & $1.003 \pm 0.149$ & $1.026 \pm 0.150$ & $23.9 \pm 3.6$ & $16.14 \pm 6.41$ & $22.18 \pm 6.36$ & $51.06 \pm 5.82$ \\
\hline GG & 11 & $1.138 \pm 0.137$ & $0.994 \pm 0.139$ & $1.011 \pm 0.132$ & $24.8 \pm 3.4$ & $14.12 \pm 5.82$ & $20.28 \pm 6.41$ & $50.97 \pm 3.21$ \\
\hline$P$-value & & 0.803 & 0.532 & 0.469 & 0.180 & 0.731 & 0.768 & 0.925 \\
\hline \multicolumn{9}{|l|}{ rs434473 } \\
\hline AA & 104 & $1.138 \pm 0.147$ & $1.001 \pm 0.139$ & $1.007 \pm 0.140$ & $23.7 \pm 3.3$ & $15.69 \pm 6.88$ & $21.66 \pm 6.92$ & $50.98 \pm 5.88$ \\
\hline$A G$ & 210 & $1.135 \pm 0.132$ & $0.999 \pm 0.144$ & $1.016 \pm 0.136$ & $23.6 \pm 3.3$ & $15.85 \pm 6.85$ & $21.74 \pm 7.22$ & $51.25 \pm 5.55$ \\
\hline GG & 86 & $1.156 \pm \pm 0.138$ & $0.987 \pm 0.144$ & $1.014 \pm 0.138$ & $23.8 \pm 3.6$ & $17.45 \pm 9.19$ & $22.57 \pm 7.42$ & $51.47 \pm 5.57$ \\
\hline$P$-value & & 0.560 & 0.519 & 0.535 & 0.874 & 0.203 & 0.608 & 0.822 \\
\hline \multicolumn{9}{|l|}{ rs312462 } \\
\hline GG & 287 & $1.145 \pm 0.140$ & $0.998 \pm 0.145$ & $1.016 \pm 0.139$ & $23.9 \pm 3.3$ & $16.93 \pm 7.61$ & $22.67 \pm 7.01$ & $51.06 \pm 5.71$ \\
\hline GA & 103 & $1.122 \pm 0.126$ & $0.985 \pm 0.135$ & $1.010 \pm 0.134$ & $23.2 \pm 3.3$ & $14.26 \pm 6.69^{*}$ & $19.62 \pm 7.01^{* *}$ & $51.70 \pm 5.52$ \\
\hline $\mathrm{AA}$ & 10 & $1.123 \pm 0.178$ & $1.107 \pm 0.117$ & $1.161 \pm 0.139$ & $22.1 \pm 3.8$ & $13.54 \pm 6.88$ & $24.10 \pm 4.36$ & $50.51 \pm 3.21$ \\
\hline$P$-value & & 0.234 & 0.254 & 0.144 & 0.632 & 0.003 & 0.001 & 0.570 \\
\hline
\end{tabular}

Abbreviations: BMD, bone mineral density; BMI, body mass index; PFM, percentage fat mass; SNP, single-nucleotide polymorphism; TFM, total fat mass; TLM, total lean mass. The $P$-values are the results of analysis of variance (ANOVA) tests for the least-square mean of the BMD among various genotypes after adjusting for the significant covariates of age, weight and height. The $P$-values are the results of ANOVA tests for the least-square mean of the BMI, TFM and TLM among various genotypes after adjusting for age as a significant covariate. Bold indicates significant $P$-values $(P<0.05)$. ${ }^{\#} P=0.001$, AA genotype versus GG genotype; ${ }^{\# \#} P=0.014$, AA genotype versus GG genotype; ${ }^{*} P=0.011$, GG genotype versus GA genotype; ${ }^{\star \star} P=0.003$, GG genotype versus $G A$ genotype. 
(including the within-family association and 1000-permutation tests) between any SNPs and the BMD at the lumbar spine and hip, although rs2073438 was associated with the femoral neck BMD in the total-population analysis $(P=0.029)$ (Table 6).

Table $6 \quad P$-values of tests for population stratification, total association and within-family association using the QTDT.

\begin{tabular}{|c|c|c|c|c|c|}
\hline & rs 2073438 & $r s 2292350$ & rs312470 & rs434473 & rs312462 \\
\hline \multicolumn{6}{|c|}{ Tests of population stratification } \\
\hline Lumbar spine BMD & 0.409 & 0.170 & 0.558 & 0.531 & 0.957 \\
\hline Femoral neck BMD & 0.335 & 0.190 & 0.783 & 0.760 & 0.923 \\
\hline Total hip BMD & 0.535 & 0.303 & 0.957 & 0.828 & 0.712 \\
\hline BMI & 0.110 & 0.569 & 0.828 & 0.894 & 0.870 \\
\hline TFM & 0.408 & 0.456 & 0.438 & 0.268 & 0.751 \\
\hline PFM & 0.377 & 0.351 & 0.457 & 0.135 & 0.421 \\
\hline TLM & 0.989 & 0.206 & 0.817 & 0.349 & 0.471 \\
\hline \multicolumn{6}{|l|}{ Test of total association } \\
\hline Lumbar spine BMD & 0.683 & 0.738 & 0.554 & 0.542 & 0.501 \\
\hline Femoral neck BMD & 0.029 & 0.172 & 0.279 & 0.081 & 0.655 \\
\hline Total hip BMD & 0.420 & 0.851 & 0.405 & 0.915 & 0.276 \\
\hline $\mathrm{BMI}$ & 0.146 & 0.669 & 0.289 & 0.369 & 0.929 \\
\hline TFM & 0.002 & 0.434 & 0.942 & 0.472 & 0.0012 \\
\hline PFM & 0.007 & 0.668 & 0.758 & 0.744 & 0.001 \\
\hline TLM & 0.331 & 0.405 & 0.853 & 0.977 & 0.284 \\
\hline \multicolumn{6}{|c|}{ Test of within-family association } \\
\hline Lumbar spine BMD & 0.359 & 0.293 & 0.845 & 0.399 & 0.814 \\
\hline Femoral neck BMD & 0.826 & 0.585 & 0.479 & 0.585 & 0.909 \\
\hline Total hip BMD & 0.892 & 0.411 & 0.700 & 0.809 & 0.844 \\
\hline $\mathrm{BMI}$ & 0.038 & 0.489 & 0.781 & 0.624 & 0.907 \\
\hline TFM & 0.007 & 0.283 & 0.608 & 0.192 & 0.049 \\
\hline PFM & 0.012 & 0.323 & 0.755 & 0.184 & 0.117 \\
\hline TLM & 0.492 & 0.669 & 0.766 & 0.474 & 0.846 \\
\hline \multicolumn{6}{|c|}{ P-value of 1000 permutations of the within-family association } \\
\hline Lumbar spine BMD & 0.344 & 0.267 & 0.812 & 0.365 & 0.770 \\
\hline Femoral neck BMD & 0.830 & 0.628 & 0.457 & 0.610 & 0.925 \\
\hline Total hip BMD & 0.887 & 0.446 & 0.705 & 0.832 & 0.801 \\
\hline BMI & 0.122 & 0.599 & 0.838 & 0.692 & 0.927 \\
\hline TFM & 0.033 & 0.385 & 0.673 & 0.294 & 0.126 \\
\hline PFM & 0.015 & 0.312 & 0.754 & 0.163 & 0.125 \\
\hline TLM & 0.153 & 0.696 & 0.793 & 0.531 & 0.854 \\
\hline
\end{tabular}

Abbreviations: BMD, bone mineral density; BMI, body mass index; PFM percentage fat mass; QTDT, quantitative transmission disequilibrium test; TFM, total fat mass; TLM, total lean mass. The BMD values are adjusted for age, height and weight. The BMI, TFM and TLM values are adjusted for age. Bold indicates significant $P$-values $(P<0.05)$.
Relationship of haplotypes and phenotypes

We conducted haplotype analyses using genotype data from all five SNPs, and four common haplotypes accounted for $91.8 \%$ of the unrelated parents. We further evaluated the association of haplotypes and the peak BMD with the obesity phenotypes using the QTDT. There were 252, 246, 164 and 146 informative families for the TDT analysis at haplotype 1 (AGAGG), haplotype 2 (GAAAG), haplotype 3 (GGGAG) and haplotype 4 (GGAGA), respectively. We failed to find any evidence of population stratification for all haplotypes $(P>0.05)$. For the total and within-family associations, a significant association was found between the most common haplotype (haplotype 1) and the TFM and PFM (both $P<0.05)$. Furthermore, haplotype 1 was still significantly associated with the PFM after 1000 permutations (corrected $P_{\mathrm{c}}=0.033$ ), but not with the TFM (corrected $\left.P_{\mathrm{c}}=0.092\right)$. The results of the haplotype 1 -association analysis are presented in Table 7.

In addition, we also investigated the association between haplotype 1 and the BMD and obesity phenotypes in 400 unrelated sons using GLM-ANOVA. The subjects carrying at least one copy of haplotype 1 had a higher TFM and PFM than non-carriers $(P=0.006$ and 0.004 , respectively). This haplotype accounted for 2.5 and $2.7 \%$ of the variation in the TFM and PFM, respectively, in this sample of younger males. However, no significant association was found between any haplotype and the BMI, TLM or peak BMD at any site (data not shown).

\section{Discussion}

To the best of our knowledge, this is the first study to investigate the possible influence of SNPs and haplotypes in the ALOX12 gene on variations in obesity-related phenotypes. Our results showed that the polymorphism rs2073438 of the $A L O X 12$ gene is significantly associated with the TFM and PFM, but not with the BMI or TLM in young men. Furthermore, haplotype-based analyses supported this significant association from the single-locus analyses. The SNP rs2073438, with a polymorphism of $\mathrm{G}>\mathrm{T}$, is located in the

Table 7 The QTDT results for the associations between haplotype 1 and phenotypic variations

\begin{tabular}{|c|c|c|c|c|}
\hline Haplotype 1 (AGAGG) & $\begin{array}{l}\text { Test of population } \\
\text { stratification }\end{array}$ & $\begin{array}{l}\text { Test of total } \\
\text { association }\end{array}$ & $\begin{array}{l}\text { Test of within-family } \\
\text { association }\end{array}$ & $\begin{array}{l}\text { P-value of } 1000 \text { permutations } \\
\text { of the within-family association }\end{array}$ \\
\hline Lumbar spine BMD & 0.508 & 0.853 & 0.502 & 0.456 \\
\hline Femoral neck BMD & 0.347 & 0.019 & 0.835 & 0.840 \\
\hline Total hip BMD & 0.630 & 0.353 & 0.909 & 0.912 \\
\hline $\mathrm{BMI}$ & 0.848 & 0.431 & 0.856 & 0.853 \\
\hline TFM & 0.892 & 0.0001 & 0.021 & 0.092 \\
\hline PFM & 0.721 & 0.003 & 0.034 & 0.033 \\
\hline TLM & 0.284 & 0.632 & 0.256 & 0.275 \\
\hline
\end{tabular}

Abbreviations: BMD, bone mineral density; BMI, body mass index; PFM, percentage fat mass; QTDT, quantitative transmission disequilibrium test; TFM, total fat mass; TLM, total lean mass. The BMD values are adjusted for age, height and weight. The BMI, TFM and TLM values are adjusted for age. Bold indicates significant $P$-values $(P<0.05)$. 
intron1/exon2 boundary of $A L O X 12$, only 639 base pairs from the $5^{\prime}$ promoter region. Moreover, based on the information from HapMap, the criterion for tagging was set at $r^{2}>0.5$, and rs2073438 as a tagging SNP captured seven additional SNPs. Therefore, the significant association of rs2073438 with the TFM and PFM suggests that the SNP itself or the region around it may be important for transcription initiation efficiency or may be in strong LD with certain functional variants influencing the expression of the ALOX12 protein. Further study is needed to clarify how this intronic SNP affects protein function. Although significant associations were observed between rs312462 and the TFM and PFM using ANOVA, we failed to find significant results in our QTDT analyses (both within-family and 1000-permutation tests). As the QTDT analysis is free of confounding population-substructure effects compared with the traditional-association approach, the results of our study should be more valuable because of the robustness of the TDT approach. ${ }^{28}$ A possible reason for our result is that the heterozygosity of rs312462 is relatively low, and the statistical power of a family-based-association study depends directly on the degree of allelic heterozygosity. ${ }^{29}$

Interestingly, we found a significant association between rs2073438 and the TFM, but not the BMI. The BMI has been widely used as a surrogate phenotype for the assessment of obesity status. However, the BMI alone may not accurately reflect the percentage of body fat and the relative contributions of muscle and fat, ${ }^{30}$ and as an index of body fatness, BMI is significantly influenced by age and sex. ${ }^{31}$ The subjects of this study were healthy young men aged 20-40 years. The TLM in our sample accounted for $\sim 70 \%$ of the body mass; we speculate that the BMI in this study may be skewed by the TLM so that the BMI values of these young Chinese men do not accurately reflect body fat mass. Our results are in accordance with the study of Liu et al., ${ }^{32}$ in which the polymorphisms of the MTHFR gene were significantly associated with the BMI; however, the association diminished after adjusting for the LM. Therefore, the FM as measured by DXA is a more refined measure of obesity.

ALOX12 belongs to the lipoxygenase superfamily, which catalyzes the insertion of molecular oxygen into polyunsaturated fatty acids. It has become increasingly clear that polyunsaturated fatty acids have an important function in the control of adipogenesis and reduce the expression of many enzymes involved in lipid metabolism. ${ }^{33,34}$ Although the exact mechanisms by which polyunsaturated fatty acids exert their effects are not completely understood, several transcription factors and nuclear receptors have been identified as critical regulators of several important genes of lipid metabolism. PPARG, a nuclear transcription factor, is a dominant regulator of adipocyte-specific genes contributing to adipocyte differentiation, susceptibility to obesity and insulin sensitivity. ${ }^{35,36}$ The substrates of ALOX12 (arachidonic acids) and the products of the lipoxygenase reaction are both effective PPARG activators, and ligand activation of PPAR $\gamma$ promotes the differentiation of mesenchymal stem cells into adipocytes. Studies have shown that ALOX12 has an important function in obesity-related diseases, such as hypertension, diabetes and atherosclerosis. ${ }^{22-24}$ More recently, Mehrabian et al. $^{37}$ showed that ALOX5, another member of the lipoxygenase superfamily, has pleiotropic effects on adiposity and type 2 diabetes-related traits. Our results, taken together with the above studies, suggest the involvement of SNP rs2073438 and the AGAGG haplotype in ALOX12 in the variation of obesity, perhaps through a PPARG-dependent pathway.

To date, $>200$ different candidate genes have been associated with obesity-related phenotypes, ${ }^{38}$ and most of those genes regulate the energy balance through the complex central nervous system. ${ }^{39}$ The mRNA and protein of ALOX12 has been described mainly in neurons and also in some glial cells throughout the cerebrum, basal ganglia and hippocampus. ${ }^{40,41}$ Recently, Pratico et al. ${ }^{42}$ showed that the protein levels and activity of ALOX12 in affected frontal and temporal regions of Alzheimer's disease brains were significantly increased. The levels of the metabolic products of ALOX12 were also markedly elevated in cerebrospinal fluid from mild cognitive impairment and Alzheimer's disease brains. $^{43}$ In these studies, they believed that ALOX12 metabolic pathway increased oxidative stress and inflammatory responses, which is likely to have a more active function in the pathogenesis of Alzheimer's disease. Obesity was determined as a state of chronic oxidative stress and oxidative stress might be a major mechanism underlying the development of obesity-related co-morbidities. ${ }^{44,45}$ We suspected that there may exist ALOX12 overexpression in central nervous system similar to Alzheimer's disease, to affect the energy balance. However, all of the above are just hypotheses and wait to be tested by further functional analyses.

Polymorphism in ALOX12 has been associated with the BMD in several independent studies. Xiong et al. ${ }^{19}$ first performed an association analysis of 20 osteoporosis candidate genes on the BMD variation at three clinically important skeletal sites: the spine, hip and ultradistal radius. Their findings show that $A L O X 12$ is a suggestive gene $(0.01<P \leqslant 0.05)$ for the BMD at the hip and ultradistal radius in the total-population sample. However, the significant association was mainly driven by the female subjects, suggesting the possibility of a sex-specific association of ALOX12 with the BMD. Extensive evidence suggests that the loci that regulate the BMD do so in a sex-specific manner in both human beings and experimental animals. ${ }^{19,46}$ The sex specificity of the associations between genes and the BMD may involve the regulation of sex steroids. In addition, Mullin et al. ${ }^{20}$ also found that three SNPs from $A L O X 12$ were significantly associated with the BMD at the spine and hip in postmenopausal Caucasian women. In this study, we studied 400 Chinese nuclear families with male offspring and used TDT to simultaneously test the linkage and association of ALOX12 gene polymorphisms with the peak BMD. No significant within-family associations were found between ALOX12 SNPs or haplotypes and the peak BMD in young 
men (aged 20-40 years). However, Ichikawa et al. ${ }^{21}$ showed evidence of an association between the spine BMD and six SNPs in the ALOX12 gene in both White men (aged 18-61 years) and women (aged 20-50 years). The conflicting results may be due to the differences between the participants, especially their ages, ethnicities or some other confounding issue. This is the first study to investigate the possible influence of $A L O X 12$ SNPs and haplotypes on the BMD variation in a non-Caucasian population. Therefore, our results should be interpreted cautiously, and further studies should be conducted to determine the strength of these associations in various populations.

We also examined the relationship between the BMD and the TFM and TLM in young men. Our results showed that both the TFM and the TLM had significant positive associations with the BMD, and the TLM was a stronger predictor of the BMD than the TFM, consistent with previous studies. ${ }^{11,47}$ However, Cui et al. ${ }^{10}$ reported that the LM was the only independent factor contributing to the BMD, and the FM was negatively related to the BMD in younger men. In contrast, other independent studies have suggested that the TFM has a stronger effect on the BMD in both pre- and post-menopausal women. ${ }^{48}$ The influence of body composition on the BMD may be confounded by gender, age, menopausal status and other environmental factors. ${ }^{49}$

Our study has several strengths. First, we chose all six tagging SNPs of the ALOX12 gene. The extensive LD between SNPs guaranteed that the entire gene had been tested for an association with the BMD and obesity phenotypes. Second, our sample was relatively large, composed of 1215 individuals from 400 nuclear families. Therefore, adequate statistical power was ensured to find the genetic variants of modest effect sizes. Third, we performed the QTDT and 1000-permutation analyses to avoid sample heterogeneity and false-positive results because of multiple tests. Our study has several limitations as well. Our nuclear families contained few sibling pairs, and no linkage was detected in this study. Environmental factors such as dietary fat intake were not considered in this study.

In conclusion, we have shown that a genetic variation (rs2073438 and haplotype AGAGG) in ALOX12 is associated with the TFM in young Chinese men, but we failed to observe a significant association between SNPs or haplotypes and the peak BMD at any site. Our findings suggest that ALOX12 is potentially important in the pathogenesis of human obesity. Although the mechanisms underlying this relationship are largely unknown, we speculate that ALOX12 may exert its effect on fat mass variation by activating the PPARG pathway or affecting central nervous system to regulate energy homeostasis.

\section{Conflict of interest}

The authors declare no conflict of interest.

\section{Acknowledgements}

The study was supported by the National Science Foundation of China (NSFC) (No. 30570891, 30771019 and 30800387) and Program of Shanghai Chief Scientist (Project No. 08XD1403000).

\section{References}

1 Zhao LJ, Jiang H, Papasian CJ, Maulik D, Drees B, Hamilton J et al. Correlation of obesity and osteoporosis: effect of fat mass on the determination of osteoporosis. J Bone Miner Res 2008; 23: 17-29.

2 Ensrud KE, Ewing SK, Stone KL, Cauley JA, Bowman PJ, Cummings SR. Intentional and unintentional weight loss increase bone loss and hip fracture risk in older women. J Am Geriatr Soc 2003; 51: 1740-1747.

3 Felson DT, Zhang Y, Hannan MT, Anderson JJ. Effects of weight and body mass index on bone mineral density in men and women: the Framingham study. J Bone Miner Res 1993; 8: 567-573.

4 Plumb MS, Aspden RM. High levels of fat and (n-6) fatty acids in cancellous bone in osteoarthritis. Lipids Health Dis 2004; 3: 12.

5 Maes HH, Neale MC, Eaves LJ. Genetic and environmental factors in relative body weight and human adiposity. Behav Genet 1997; 27: 325-351.

6 Peacock M, Turner CH, Econs MJ, Foroud T. Genetics of osteoporosis. Endocr Rev 2002; 23: 303-326.

7 Deng FY, Lei SF, Li MX, Jiang C, Dvornyk V, Deng HW. Genetic determination and correlation of body mass index and bone mineral density at the spine and hip in Chinese Han ethnicity. Osteoporos Int 2006; 17: 119-124.

8 Heim M, Frank O, Kampmann G, Sochocky N, Pennimpede T, Fuchs $P$ et al. The phytoestrogen genistein enhances osteogenesis and represses adipogenic differentiation of human primary bone marrow stromal cells. Endocrinology 2004; 145: 848-859.

9 Rosen CJ, Bouxsein ML. Mechanisms of disease: is osteoporosis the obesity of bone? Nat Clin Pract Rheumatol 2006; 2: 35-43.

10 Cui LH, Shin MH, Kweon SS, Park KS, Lee YH, Chung EK et al. Relative contribution of body composition to bone mineral density at different sites in men and women of South Korea. J Bone Miner Metab 2007; 25: 165-171.

11 Wang MC, Bachrach LK, Van Loan M, Hudes M, Flegal KM, Crawford $\mathrm{PB}$. The relative contributions of lean tissue mass and fat mass to bone density in young women. Bone 2005; 37: 474-481.

12 Gjesdal CG, Halse JI, Eide GE, Brun JG, Tell GS. Impact of lean mass and fat mass on bone mineral density: The Hordaland Health Study. Maturitas 2008; 59: 191-200.

13 Tang ZH, Xiao P, Lei SF, Deng FY, Zhao LJ, Deng HY et al. A bivariate whole-genome linkage scan suggests several shared genomic regions for obesity and osteoporosis. J Clin Endocr Metab 2007; 92: 2751-2757.

14 Reich MS, Jarvis JP, Silva MJ, Cheverud JM. Genetic relationships between obesity and osteoporosis in LGXSM recombinant inbred mice. Genet Res 2008; 90: 433-444.

15 Klein RF, Allard J, Avnur Z, Nikocheva T, Rotstein D, Carlos AS et al. Regulation of bone mass in mice by the lipoxygenase gene Alox15. Science 2004; 303: 229-232.

16 Serhan CN, Jain A, Marleau S, Clish C, Kantarci A, Behbehani B et al. Reduced inflammation and tissue damage in transgenic rabbits overexpressing 15-lipoxygenase and endogenous antiinflammatory lipid mediators. I Immunol 2003; 171: 6856-6865.

17 Chen XS, Kurre U, Jenkins NA, Copeland NG, Funk CD. cDNA cloning, expression, mutagenesis of C-terminal isoleucine, genomic structure, and chromosomal localizations of murine 12-lipoxygenases. J Biol Chem 1994; 269: 13979-13987. 
18 Kühn H, Barnett J, Grunberger D, Baecker P, Chow J, Nguyen B et al. Overexpression, purification and characterization of human recombinant 15-lipoxygenase. Biochim Biophys Acta 1993; 1169: 80-89.

19 Xiong DH, Shen H, Zhao LJ, Xiao P, Yang TL, Guo Y et al. Robust and comprehensive analysis of 20 osteoporosis candidate genes by very high-density single-nucleotide polymorphism screen among 405 white nuclear families identified significant association and gene-gene interaction. J Bone Miner Res 2006; 21 : 1678-1695.

20 Mullin BH, Spector TD, Curtis CC, Ong GN, Hart DJ, Hakim AJ et al. Polymorphisms in ALOX12, but not ALOX15, are significantly associated with BMD in postmenopausal women. Calcif Tissue Int 2007; 81: 10-17.

21 Ichikawa S, Koller DL, Johnson ML, Lai D, Xuei X, Edenberg HJ et al. Human ALOX12, but not ALOX15, is associated with BMD in white men and women. J Bone Miner Res 2006; 21: 556-564.

22 Bleich D, Chen S, Zipser B, Sun D, Funk CD, Nadler JL. Resistance to type 1 diabetes induction in 12-lipoxygenase knockout mice. J Clin Invest 1999; 103: 1431-1436.

23 Huo Y, Zhao L, Hyman MC, Harry BL, Burcin T, Forlow SB et al. Critical role of macrophage 12/15-lipoxygenase for atherosclerosis in apolipoprotein E-deficient mice. Circulation 2004; 110: 2024-2031.

24 Quintana LF, Guzman B, Collado S, Claria J, Poch E. A coding polymorphism in the 12-lipoxygenase gene is associated to essential hypertension and urinary 12(S)-HETE. Kidney Int 2006; 69: 526-530.

25 Barrett JC, Fry B, Maller J, Daly MJ. Haploview: analysis and visualization of LD and haplotype maps. Bioinformatics 2005; 21: 263-265.

26 Zhang ZL, He JW, Qin YJ, Hu YQ, Li M, Liu YJ et al. Association between SNP and haplotypes in PPARGC1 and adiponectin genes and bone mineral density in Chinese nuclear families. Acta Pharmacol Sin 2007; 28: 287-295.

27 Zhang ZL, He JW, Qin YJ, Hu YQ, Li M, Zhang H et al. Association between myostatin gene polymorphisms and peak BMD variation in Chinese nuclear families. Osteoporos Int 2008; 19: 39-47.

28 Abecasis GR, Cookson WO, Cardon LR. Pedigree tests of transmission disequilibrium. Eur J Hum Genet 2000; 8: 545-551.

29 Liu YJ, Liu PY, Long J, Lu Y, Elze L, Recker RR et al. Linkage and association analyses of the UCP3 gene with obesity phenotypes in Caucasian families. Physiol Genomics 2005; 22: 197-203.

30 Ode JJ, Pivarnik JM, Reeves MJ, Knous JL. Body mass index as a predictor of percent fat in college athletes and nonathletes. Med Sci Sport Exer 2007; 39: 403-409.

31 Flegal KM, Shepherd JA, Looker AC, Graubard BI, Borrud LG, Ogden CL et al. Comparisons of percentage body fat, body mass index, waist circumference, and waist-stature ratio in adults. Am J Clin Nutr 2009; 89: 500-508.

32 Liu X, Zhao LJ, Liu YJ, Xiong DH, Recker RR, Deng HW. The MTHFR gene polymorphism is associated with lean body mass but not fat body mass. Hum Genet 2008; 123: 189-196.

33 Sampath H, Ntambi JM. Polyunsaturated fatty acid regulation of genes of lipid metabolism. Ann Rev Nutr 2005; 25: 317-340.

34 Clarke SD. Polyunsaturated fatty acid regulation of gene transcription: a molecular mechanism to improve the metabolic syndrome. J Nutr 2001; 131: 1129-1132.
35 Kadowaki T, Hara K, Yamauchi T, Terauchi Y, Tobe K, Nagai R. Molecular mechanism of insulin resistance and obesity. Exp Bio Med 2003; 228: 1111-1117.

36 Barroso I, Gurnell M, Crowley VE, Agostini M, Schwabe JW, Soos MA et al. Dominant negative mutations in human PPARgamma associated with severe insulin resistance, diabetes mellitus and hypertension. Nature 1999; 402: 880-883.

37 Mehrabian M, Schulthess FT, Nebohacova M, Castellani LW, Zhou Z, Hartiala J et al. Identification of ALOX5 as a gene regulating adiposity and pancreatic function. Diabetologia 2008; 51: 978-988.

38 Perusse L, Rankinen T, Zuberi A, Chagnon YC, Weisnagel SJ, Argyropoulos G et al. The human obesity gene map: the 2004 update. Obes Res 2005; 13: 381-490.

39 Laclaustra M, Corella D, Ordovas JM. Metabolic syndrome pathophysiology: the role of adipose tissue. Nutr Metab Cardiovasc Dis 2007; 17: 125-139.

40 Nishiyama M, Watanabe T, Ueda N, Tsukamoto $\mathrm{H}$, Watanabe $\mathrm{K}$. Arachidonate 12-lipoxygenase is localized in neurons, glial cells, and endothelial cells of the canine brain. J Histochem Cytochem 1993; 41: 111-117.

41 Nishiyama M, Okamoto $\mathrm{H}$, Watanabe T, Hori T, Hada T, Ueda N et al. Localization of arachidonate 12-lipoxygenase in canine brain tissues. J Neurochem 1992; 58: 1395-1400.

42 Pratico D, Zhukareva V, Yao Y, Uryu K, Funk CD, Lawson JA et al. 12/15-lipoxygenase is increased in Alzheimer's disease: possible involvement in brain oxidative stress. Am J Pathol 2004; 164 1655-1662.

43 Yao Y, Clark CM, Trojanowski JQ, Lee VM, Praticò D. Elevation of 12/15 lipoxygenase products in AD and mild cognitive impairment. Ann Neurol 2005; 58: 623-626.

44 Holguin F, Fitzpatrick A. Obesity, asthma, and oxidative stress. J Appl Physiol 2010; 108: 754-759.

45 Hopps E, Noto D, Caimi G, Averna MR. A novel component of the metabolic syndrome: the oxidative stress. Nutr Metab Cardiovasc Dis 2010; 20: 72-77.

46 Ioannidis JP, Ng MY, Sham PC, Zintzaras E, Lewis CM, Deng HW et al. Meta-analysis of genome-wide scans provides evidence for sex- and site-specific regulation of bone mass. J Bone Miner Res 2007; 22: 173-183.

47 Young D, Hopper JL, Macinnis RJ, Nowson CA, Hoang $\mathrm{NH}$ Wark JD. Changes in body composition as determinants of longitudinal changes in bone mineral measures in 8 to 26-year-old female twins. Osteoporos Int 2001; 12: 506-515.

48 Reid IR. Relationships among body mass, its components, and bone. Bone 2002; 31: 547-555.

49 Hsu YH, Venners SA, Terwedow HA, Feng Y, Niu T, Li Z et al. Relation of body composition, fat mass, and serum lipids to osteoporotic fractures and bone mineral density in Chinese men and women. Am J Clin Nutr 2006; 83: 146-154.

\section{(c)}

This work is licensed under the Creative Commons Attribution-NonCommercial-No Derivative Works 3.0 Unported License. To view a copy of this license, visit http://creativecommons.org/ licenses/by-nc-nd/3.0/ 